\title{
Influence of Statins on LDL- and HDL-Cholesterol and Plasma Fatty Acids in Elderly Japanese Ischemic Stroke Patients
}

\author{
Mori T*, Tanno Y, Kasakura S, Yoshioka K and \\ Nakai $\mathbf{N}$ \\ Department of Stroke Treatment, Shonan Kamakura \\ General Hospital Stroke Center, Japan \\ *Corresponding author: Takahisa Mori, Department \\ of Stroke Treatment, Shonan Kamakura General \\ Hospital Stroke Center, Okamoto 1370-1, Kamakura City, \\ Kanagawa 247-8533, Japan
}

Received: May 07, 2017; Accepted: June 01, 2017; Published: June 09, 2017

\begin{abstract}
Introduction: Stroke sometimes occurs in elderly patients who are treated with statins. The aim of our retrospective study was to investigate what influences statins had on plasma levels of LDL-C, HDL-C, triglyceride (TG) and fatty acids (FA), particularly essential FAs, in elderly Japanese acute ischemic stroke patients.
\end{abstract}

Materials and Methods: We conducted a cross-sectional study of Japanese acute ischemic stroke patients aged between 50 and 74 years admitted to our institution between Sep 2015 and Aug 2016 within 24 hours of stroke onset who took blood examination for plasma lipid levels of LDL-C, HDL-C, TG and FAs such as palmitic acid $(\mathrm{PaA})$, stearic acid $(\mathrm{StA})$, oleic acid $(\mathrm{OIA})$, linoleic acid (LiA), dihomo-gamma-linolenic acid (DHLA), arachidonic acid (AA), eicosapentaenoic acid (EPA) and docosahexaenoic acid (DHA). We assessed plasma lipid levels in patients taking statins (group S) and in patients not taking statins (group NS).

Results: One hundred forty-seven patients matched our criteria. Average age was 68 years. On arrival to the hospital, 30 patients took statins (group S) and 117 didn't (group NS). In group S and NS, LDL-C was 84.6 and $130.1 \mathrm{mg} / \mathrm{dl}$ $(p<0.0001), H D L-C$ was 50.5 and $60.3 \mathrm{mg} / \mathrm{dl}(p<0.01)$, TG was 196.8 and 135.7 $\mathrm{mg} / \mathrm{dl}(\mathrm{p}<0.05)$. There was no difference in plasma levels of any FAs except LiA (722.5 vs. $880.1 \mu \mathrm{g} / \mathrm{mL},(p<0.001))$.

Conclusion: Plasma levels of LDL-C, HDL-C and LiA were lower in group $S$; however there were no differences in PaA, StA, OIA, DHLA, AA, EPA or DHA.

Keywords: Ischemic stroke; LDL-C; HDL-C

\section{Abbreviations}

LDL: Low Density Lipoprotein; LDL-C: Low Density Lipoprotein Cholesterol; HDL: High Density Lipoprotein; HDL-C: High Density Lipoprotein Cholesterol; TG: Triglyceride; PaA: Palmitic Acid; StA: Stearic Acid; OlA: Oleic Acid; LiA: Linoleic Acid; DHLA: Dihomo-Gamma-Linolenic Acid; AA: Arachidonic Acid; EPA: Eicosapentaenoic Acid; DHA: Docosahexaenoic Acid; FA: Fatty Acid; SFA: Saturated FA; PUFA: Polyunsaturated Fatty Acid; BMI: Body Mass Index; NIHSS: National Institute of Health Stroke Scale

\section{Introduction}

Previous statin trials have reported that the relative risk reduction (RRR) for stroke as a secondary endpoint was $21 \%$ with no heterogeneity between trials [1] in patients with high LDL-C level. A previous study reported that $80 \mathrm{mg}$ of atorvastatin per day reduced the overall incidence of strokes and of cardiovascular events, despite a small increase in the incidence of hemorrhagic stroke [2]. Subanalysis of previous studies reported that administration of highly purified eicosapentaenoic acid (EPA) appeared to reduce the risk of recurrent stroke in a Japanese population of hypercholesterolemic patients receiving low-dose statin therapy $[3,4]$. Indeed, statins are very commonly used against dyslipidemia in elderly people aged between 50 and 74 years, however, ischemic stroke sometimes occurs in them despite oral statins. Fatty acids (FAs) are significant components of LDL, HDL and TG and it remains unknown how stains influence plasma FA levels; i.e., saturated fatty acids (SFAs) and polyunsaturated fatty acids (PUFAs) level. The aim of our retrospective study was to investigate what influences statins had on plasma levels of not only LDL-C, HDL-C and TG but also fatty acids (FAs), particularly essential FAs such as linoleic acid (LiA) of n-6 PUFAs and EPA of n-3 PUFAs, in elderly Japanese ischemic stroke patients at the onset of stroke.

\section{Materials and Methods}

\section{Study design and subjects}

We conducted a cross-sectional study of Japanese acute stroke patients aged between 50 and 74 years, who were admitted to our institution between Sep 2015 and Aug 2016 within 24 hours of stroke onset and who took blood examination for plasma lipid levels.

\section{Exclusion}

We excluded from our analysis patients who took n-3 polyunsaturated (PU) FA drugs, fibrates, or ezetimibe.

\section{Variables}

Variable we examined were plasma lipid levels of LDL-C, HDL-C,
Austin J Cerebrovasc Dis \& Stroke - Volume 4 Issue 3 - 2017 ISSN : 2381-9103 | www.austinpublishing group.com Mori et al. () All rights are reserved
Citation: Mori T, Tanno Y, Kasakura S, Yoshioka K and Nakai N. Influence of Statins on LDL- and HDLCholesterol and Plasma Fatty Acids in Elderly Japanese Ischemic Stroke Patients. Austin J Cerebrovasc Dis \& Stroke. 2017; 4(3): 1062. 
Table 1: Patients' baseline characteristics in group S and NS.

\begin{tabular}{|l|c|c|c|}
\hline & Group S & Group NS & $\mathbf{p}$ \\
\hline Number $(\mathrm{n})$ & 30 & 117 & \\
\hline Age (median, IQR) years & $68.5(65-73)$ & $68(61-72)$ & $\mathrm{ns}$ \\
\hline Male gender & $22(73 \%)$ & $75(64 \%)$ & $\mathrm{ns}$ \\
\hline BMI (mean \pm SD) kg/m2 & $24.3 \pm 0.8$ & $23.5 \pm 0.4$ & $\mathrm{~ns}$ \\
\hline Blood pressure (mean $\pm \mathrm{SD}) \mathrm{mmHg}$ & $109.4 \pm 3.6$ & $118.7 \pm 1.8$ & $<0.05$ \\
\hline NIHSS (median, IQR) & $2(1-3.25)$ & $2(1-5.5)$ & $\mathrm{ns}$ \\
\hline
\end{tabular}

Table 2: Plasma lipid levels and oral statins in group $S$ ans NS.

\begin{tabular}{|c|c|c|c|c|c|}
\hline & $\begin{array}{l}\text { Group S } \\
(n=30)\end{array}$ & & $\begin{array}{c}\text { Group NS } \\
(n=117)\end{array}$ & & p \\
\hline $\begin{array}{l}\text { LDL-C (mg/dl) (average } \\
\pm \mathrm{SD})\end{array}$ & 84.6 & -7.8 & $130.1 \pm 4.0$ & & $p<0.0001$ \\
\hline $\begin{array}{l}\mathrm{HDL}-\mathrm{C}(\mathrm{mg} / \mathrm{dl}) \text { (average } \\
\pm \mathrm{SD})\end{array}$ & 50.8 & -3.1 & 60.3 & -1.6 & $p<0.01$ \\
\hline $\begin{array}{l}\text { TG (mg/dl) (average } \\
\pm \text { SD) }\end{array}$ & 196.8 & -21.4 & 135.8 & -10.8 & $p<0.05$ \\
\hline $\begin{array}{l}\mathrm{PaA}(\mu \mathrm{g} / \mathrm{mL}) \text { (average } \\
\pm \mathrm{SD})\end{array}$ & 715.9 & -42.8 & 781.5 & -21.7 & ns \\
\hline $\begin{array}{l}\text { StA }(\mu \mathrm{g} / \mathrm{mL}) \text { (average } \\
\pm \mathrm{SD})\end{array}$ & 200.3 & -11.4 & $222.7 \pm 5.8$ & & 0.08 \\
\hline $\begin{array}{l}\text { OIA }(\mu \mathrm{g} / \mathrm{mL}) \text { (average } \\
\pm \mathrm{SD})\end{array}$ & 709.4 & -46.9 & $723 \pm 23.7$ & & ns \\
\hline $\begin{array}{l}\mathrm{LiA}(\mu \mathrm{g} / \mathrm{mL}) \text { (average } \\
\pm \mathrm{SD})\end{array}$ & 722.5 & -38.7 & 880.1 & -19.6 & $p<0.001$ \\
\hline $\begin{array}{l}\mathrm{DHLA}(\mu \mathrm{g} / \mathrm{mL}) \text { (average } \\
\pm \mathrm{SD})\end{array}$ & 29.6 & -2 & 33.1 & -1 & ns \\
\hline $\begin{array}{l}\mathrm{AA}(\mu \mathrm{g} / \mathrm{mL}) \text { (average } \\
\pm \mathrm{SD})\end{array}$ & $166.7 \pm 8.4$ & & $166 \pm 4.2$ & & ns \\
\hline $\begin{array}{l}\text { EPA }(\mu \mathrm{g} / \mathrm{mL}) \text { (average } \\
\pm \mathrm{SD})\end{array}$ & 76.8 & -7 & $68 \pm 3.5$ & & ns \\
\hline $\begin{array}{l}\mathrm{DHA}(\mu \mathrm{g} / \mathrm{mL}) \text { (average } \\
\pm \mathrm{SD})\end{array}$ & $129.2 \pm 8.1$ & & $136.5 \pm 4.1$ & & ns \\
\hline \multirow[t]{4}{*}{ Stains } & $\begin{array}{l}\text { Rosuvastatin; } \\
9\end{array}$ & & none & & \\
\hline & $\begin{array}{c}\text { Pitavastatin; } \\
9\end{array}$ & & & & \\
\hline & $\begin{array}{c}\text { Atorvastatin; } \\
6\end{array}$ & & & & \\
\hline & Pravastatin; 6 & & & & \\
\hline
\end{tabular}

TG and FAs such as palmitic acid $(\mathrm{PaA})$ and stearic acid (StA) of SFAs, oleic acid (OlA) of n-9 PUFA, LiA, dihomo-gamma-linolenic acid (DHLA) and arachidonic acid (AA) of n-6 PUFA, EPA and docosahexaenoic acid (DHA) of n-3 PUFA.

\section{Evaluation}

We assessed baseline patients' characteristics such as age, gender, BMI and NIHSS, and plasma lipid levels in patients taking statins (group S) and in patients not taking statins (group NS).

\section{Statistical analysis}

Age and NIHSS were expressed as median and interquartile range (IQR) and compared by Mann-Whitney's U test. Other continuous variables, expressed as mean $\pm \mathrm{SD}$, were compared by unpaired Student's t test. Categorical data were compared by using the Chi square test or the Fisher exact test. A probability of less than $\mathrm{P}=0.05$ was considered statistically significant. The JMP (version 13.0.0) program was used to perform the statistical analysis.

\section{Results}

During study period, 167 acute ischemic stroke patients aged between 50 and 74 years were admitted to our institution and 13 patients were excluded from analysis because of no blood examination of plasma FAs and subsequently 7 patients were excluded because of oral n-3 PUFA drugs or ezetimibe. Finally, one hundred fortyseven patients matched our criteria and were analyzed. Their age (median, IQR) was 68 years (63-72), male gender was 97 (66\%), their BMI (mean $\pm \mathrm{SD}$ ) was $23.7 \pm 4.3 \mathrm{~kg} / \mathrm{m} 2$, blood pressure was 116.8 $\pm 19.8 \mathrm{mmHg}$, NIHSS (median, IQR) was 2 (1-5). As standard lipid examination, LDL-C, HDL-C and TG were $120.8 \pm 46.5$, 58.3 \pm 17.3 and $148.2 \pm 119.2 \mathrm{mg} / \mathrm{dl}$. As SFAs, PaA and StA were $768.1 \pm 235.1$ and $218.1 \pm 62.9 \mu \mathrm{g} / \mathrm{mL}$. As n-9 PUFA, OlA was $720.2 \pm 255.8 \mu \mathrm{g} / \mathrm{mL}$. As n-6 PUFAs, LiA, DHLA and AA were $848.0 \pm 220.7,32.4 \pm 11.3$ and $166.2 \pm 45.6 \mu \mathrm{g} / \mathrm{mL}$. As n-3 PUFAs, EPA and DHA were $69.8 \pm$ 38.2 and $135.0 \pm 44.2 \mu \mathrm{g} / \mathrm{mL}$. Their average EPA level wasn't high. On arrival to the hospital, 30 patients (20.4\%) took statins (group S) and 117 didn't (group NS). There were no differences of patients' baseline characteristics between group S and NS except blood pressure (Table 1). In group $S$, strong (3rd generation) stains was used in $80 \%$ (Table 2). In group $S$ and NS (Table 2), LDL-C was 84.6 and $130.1 \mathrm{mg} / \mathrm{dl}$ $(\mathrm{p}<0.001)$, HDL-C was 50.5 and $60.3 \mathrm{mg} / \mathrm{dl}(\mathrm{p}<0.01)$, TG was 196.8 and $135.7 \mathrm{mg} / \mathrm{dl}(\mathrm{p}<0.05), \mathrm{PaA}$ was 715.9 and $781.5 \mu \mathrm{g} / \mathrm{mL}$ (ns), StA was 200.3 and $222.7 \mu \mathrm{g} / \mathrm{mL}(\mathrm{ns})$, OlA was 709.4 and $723.0 \mu \mathrm{g} / \mathrm{mL}$ (ns), LiA was 722.5 and $880.1 \mu \mathrm{g} / \mathrm{mL}(\mathrm{p}<0.001)$, DHLA was 29.6 and 33.1 $\mu \mathrm{g} / \mathrm{mL}(\mathrm{n}), \mathrm{AA}$ was 166.7 and $166.0 \mu \mathrm{g} / \mathrm{mL}$ (ns), EPA was 76.8 and $68.0 \mu \mathrm{g} / \mathrm{mL}$ (ns), DHA was 129.2 and $136.5 \mu \mathrm{g} / \mathrm{mL}$ (ns). There were no differences in plasma levels of any FAs except LiA in both groups. There were no differences of SFAs nor essential FAs except LiA level between two groups. In both groups, EPA level wasn't high and AA level wasn't low.

\section{Discussion}

Our results demonstrated that EPA level wasn't high in patients with nor without statin and that there were no differences of any FAs including EPA except LiA in both groups, although LDL-C and HDL-C level was lower in patients with oral statin.

Previous statin trials have reported that the relative risk reduction (RRR) for stroke as a secondary endpoint was $21 \%$ with no heterogeneity between trials [1]. Each $10 \%$ reduction in LDL-C was estimated to reduce the risk of all strokes by $13.2 \%$ (95\% CI: 4.820.6) [1]. Statins have not been shown to prevent recurrent stroke in patients with prior stroke, although a previous study reported that, in patients with recent stroke or TIA and without known coronary heart disease, $80 \mathrm{mg}$ of atorvastatin per day reduced the overall incidence of strokes and of cardiovascular events, despite a small increase in the incidence of hemorrhagic stroke [2].

Fatty acids have relation to cardiovascular risks [3,5-7]. Subanalysis of previous studies reported that administration of highly purified eicosapentaenoic acid (EPA) appeared to reduce the risk of recurrent stroke in a Japanese population of hypercholesterolemic patients receiving low-dose statin therapy $[3,4]$.

Our results demonstrated that statins didn't have influences on plasma levels of SFA nor PUFA including EPA, although cholesterol synthesis was significantly impaired. Lower LDL-C level in group S indicated that patients in group $S$ really had taken statins prior to stroke onset. It may be why statins haven't been clearly shown to prevent stroke primarily or secondarily. Essential FAs people can't 
synthesize are driven from diet. Therefore, there were no differences in essential FAs levels between patients with and without statins, and this was because diet before stroke onset may be almost the same in both groups. To prevent stroke primarily or secondarily, diet should be changed or improved in viewpoints of essential FAs.

The present study has several important limitations. The investigation was conducted in a single department at a single comprehensive stroke center, and the number of patients was small. Patients on statins prior to their stroke might be inherently different from patients not on statins prior to their stroke, because the present study was not a case-control but a retrospective cross-sectional study and it was unknown why patients didn't take statins prior to their stroke. Indeed, stain effects on essential FAs are not well known, but statins alone couldn't increase EPA level [3,4]. Furthermore, statin adherence prior to stroke onset wasn't measured. Therefore, the present study can't demonstrate statin effects or influences on essential FAs, particularly EPA of n-3 PUFA, however statin probably can't increase nor decrease EPA but also increase nor decrease AA.

\section{Conclusion}

Plasma levels of LDL-C, HDL-C and LiA were lower in group S; however there were no differences in plasma levels of PaA and StA of SFAs, OlA of n-9 PUFA, DHLA and AA except LiA of n-6 PUFA of essential FAs, EPA and DHA of n-3 PUFA of essential FAs.

\section{References}

1. Amarenco P, Labreuche J, Lavallee P, Touboul PJ. Statins in stroke prevention and carotid atherosclerosis: systematic review and up-to-date meta-analysis. Stroke. 2004; 35: 2902-2909.
2. Amarenco P, Bogousslavsky J, Callahan A, Goldstein LB, Hennerici M Rudolph $\mathrm{AE}$, et al. High-dose atorvastatin after stroke or transient ischemic attack. N Engl J Med. 2006; 355: 549-559.

3. Yokoyama $M$, Origasa $H$, Matsuzaki $M$, Matsuzawa $Y$, Saito $Y$, Ishikawa $Y$, et al. Effects of eicosapentaenoic acid on major coronary events in hypercholesterolaemic patients (JELIS): a randomised open-label, blinded endpoint analysis. Lancet. 2007; 369: 1090-1098.

4. Tanaka K, Ishikawa $Y$, Yokoyama M, Origasa H, Matsuzaki M, Saito $Y$, et al. Reduction in the recurrence of stroke by eicosapentaenoic acid for hypercholesterolemic patients: sub analysis of the JELIS trial. Stroke. 2008; 39: 2052-2058.

5. Bersch-Ferreira AC, Sampaio GR, Gehringer MO, Ross-Fernandes MB, Kovacs $\mathrm{C}$, Alves $\mathrm{R}$, et al. Association between polyunsaturated fatty acids and inflammatory markers in patients in secondary prevention of cardiovascular disease. Nutrition. 2017; 37: 30-36.

6. Del Gobbo LC, Imamura F, Aslibekyan S, Marklund M, Virtanen JK, Wennberg M, et al. $\omega-3$ Polyunsaturated Fatty Acid Biomarkers and Coronary Heart Disease: Pooling Project of 19 Cohort Studies. JAMA Intern Med. 2016; 176: 1155-1166.

7. Yang LG, Song ZX, Yin H, Yan Yan Wang, Guo Fang Shu, Hui Xia Lu, et al. Low n-6/n-3 PUFA Ratio Improves Lipid Metabolism, Inflammation, Oxidative Stress and Endothelial Function in Rats Using Plant Oils as n-3 Fatty Acid Source. Lipids. 2016; 51: 49-59.
Austin J Cerebrovasc Dis \& Stroke - Volume 4 Issue 3 - 2017 ISSN : 2381-9103 | www.austinpublishinggroup.com Mori et al. (C) All rights are reserved
Citation: Mori T, Tanno Y, Kasakura S, Yoshioka K and Nakai N. Influence of Statins on LDL- and HDLCholesterol and Plasma Fatty Acids in Elderly Japanese Ischemic Stroke Patients. Austin J Cerebrovasc Dis \& Stroke. 2017; 4(3): 1062. 